\title{
Discrimination of aminobiphenyl isomers in gas phase and investigation of their complex conformations
}

Fangling $\mathrm{Wu}^{1}$, Shutong Yang ${ }^{1}$, Xinhua $\mathrm{Da}^{2}$, Liancheng $\mathrm{Gu}^{1}$, Fuxing $\mathrm{Xu}^{1}$, Xiang Fang*2, Shaoning $\mathrm{Yu}^{* 1}$, Chuan-Fan Ding*1

1. Zhejiang Provincial Key Laboratory of Advanced Mass Spectrometry and Molecular Analysis, Institute of Mass Spectrometry, School of Material Science and Chemical Engineering, Ningbo University, Ningbo, Zhejiang 315211, China.

2. National Institute of Metrology, Beijing, 100084, China

\section{*Corresponding author:}

Xiang Fang: fangxiang@nim.ac.cn

Shaoning Yu: yushaoling@nbu.edu.cn

Prof. Chuan-Fan Ding, dingchuanfan@nbu.edu.cn

Tel \& Fax: +86-0574-87605710, 

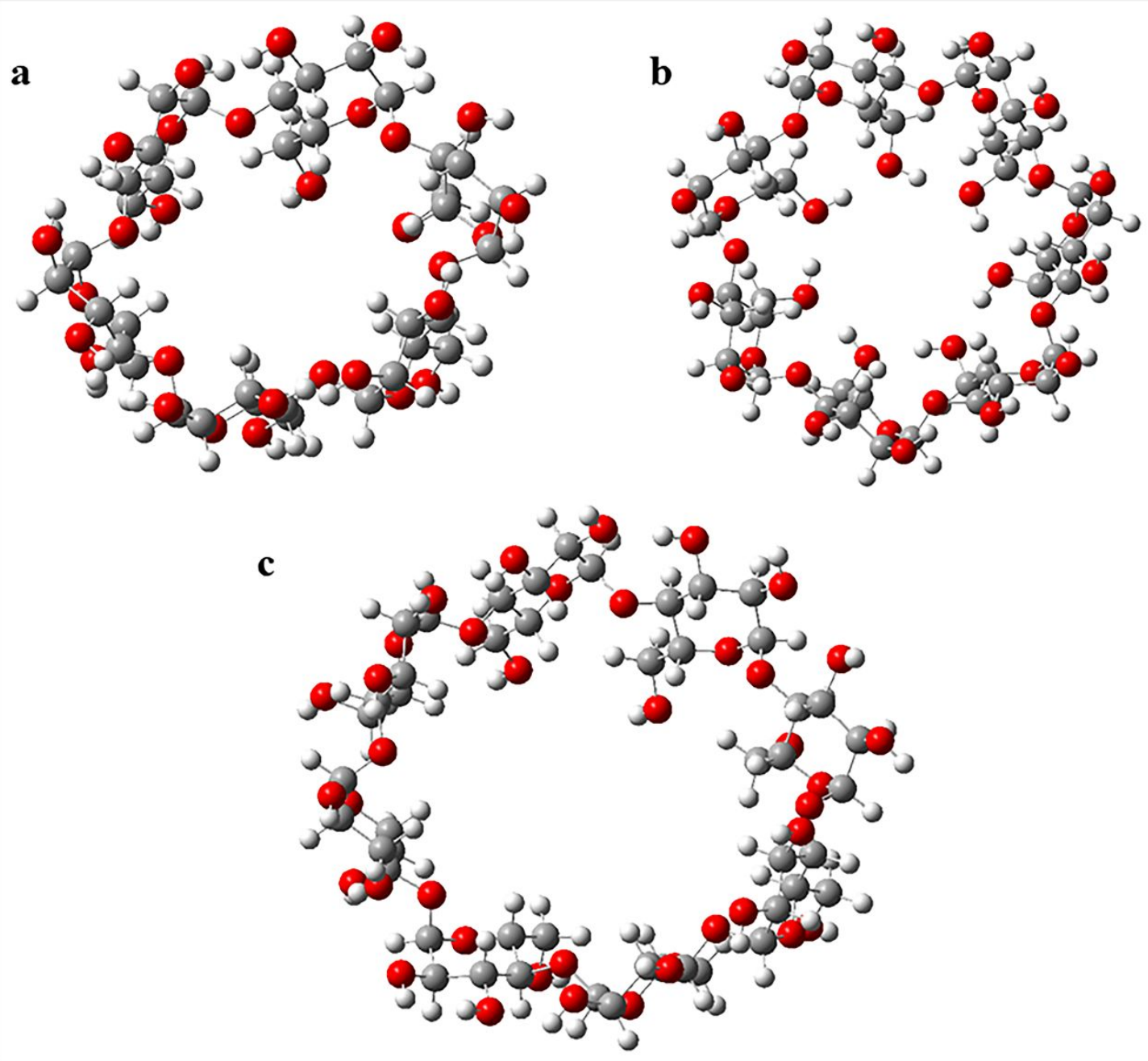

Figure S1 Structure for the CDs (a) $\alpha-C D$; (b) $\beta-C D$; (c) $\gamma-C D$

\section{Confirming the formation of the $[\mathrm{CD}+\mathrm{ABP}+\mathrm{H}]^{+}$}

To further confirm the formation of the stable inclusion complex, the ESI mass spectra for the mixture of $\alpha, \beta$ or $\gamma$-CD with $\mathrm{ABP}$ in different molar ratios are shown in Fig. S2. It is seen that no obvious peak for other reaction products are detected except for the peaks of $[\alpha-\mathrm{CD}+\mathrm{ABP}+\mathrm{H}]^{+},[\beta-\mathrm{CD}+\mathrm{ABP}+\mathrm{H}]^{+},[\gamma-\mathrm{CD}+\mathrm{ABP}+\mathrm{H}]^{+}$(the $1: 1$ inclusion complexes). Meanwhile, it is observed that when different molar ratios of the reactants were mixed, the coordination number of the resulting product was fixed at $1: 1$, which implies the coordination number of the final product is independent of the initial molar ratios of the reactants. Meanwhile, the different incubation times of $0,5,30$, and $60 \mathrm{~min}$ for the mixture of 1:1 molar ratio $\mathrm{CD}$ and 
ABP were further studied. As shown in Fig. S3, the detected mass spectra are still the $1: 1$ inclusion complexes of $[\alpha-\mathrm{CD}+\mathrm{ABP}+\mathrm{H}]^{+},[\beta-\mathrm{CD}+\mathrm{ABP}+\mathrm{H}]^{+},[\gamma-\mathrm{CD}+\mathrm{ABP}+\mathrm{H}]^{+}$. Based on this, it infers that the reaction product is not a cluster formed by ions or molecules, but on the nonspecific binding interaction of $[\mathrm{CD}+\mathrm{ABP}+\mathrm{H}]^{+}$complex.
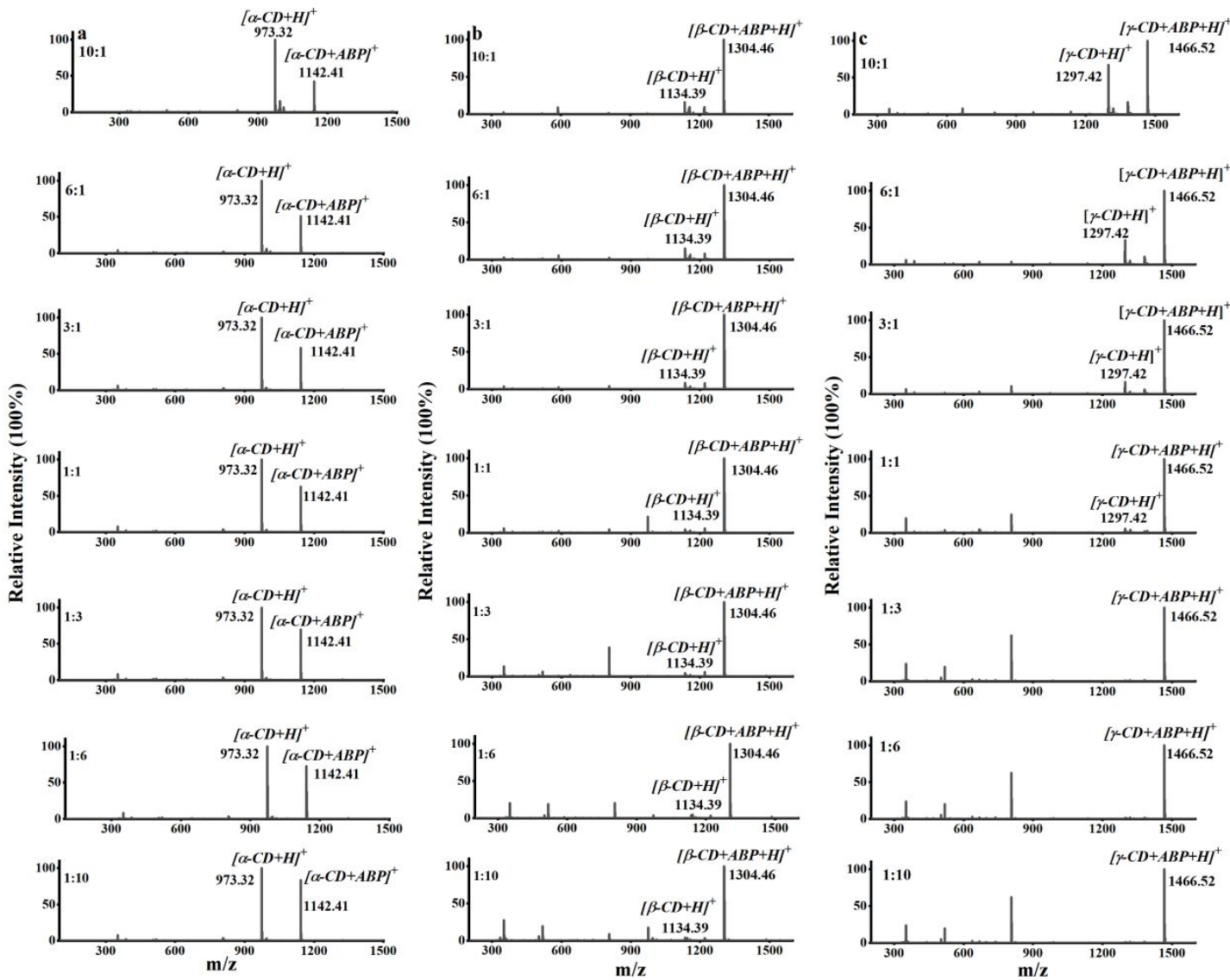

Figure S2 The mass spectra obtained by the different molar ratios for CD in complex with $\mathrm{ABP}$ (a) $\alpha-\mathrm{CD}$; (b) $\beta-\mathrm{CD}$; and (c) $\gamma-\mathrm{CD}$ 

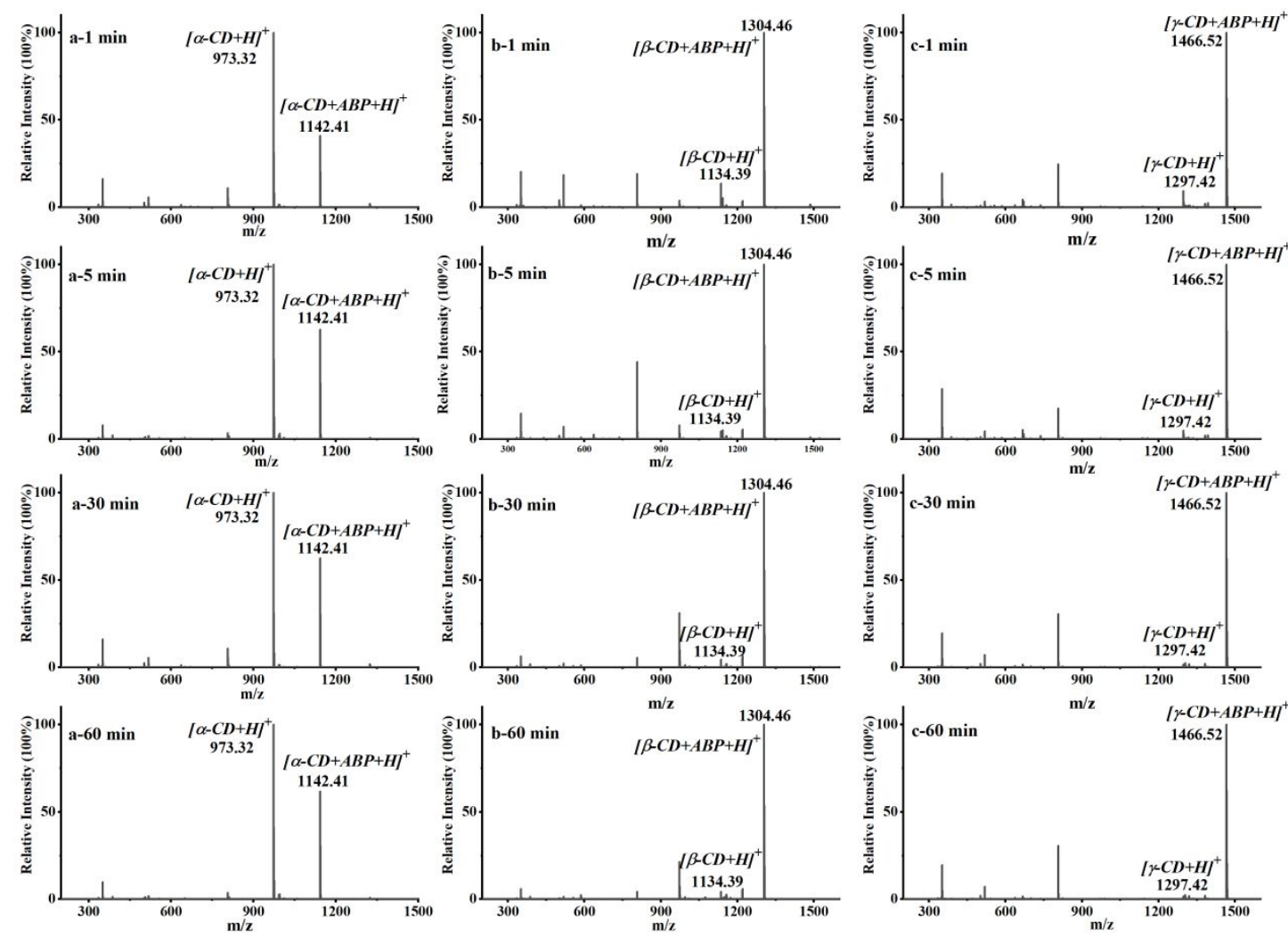

Figure S3 The mass spectra obtained by the different incubation times of 0, 5, 30 and 60 min for $\mathrm{CD}$ in complex with $\mathrm{ABP}$ (a) $\alpha-\mathrm{CD}$; (b) $\beta-\mathrm{CD}$; and (c) $\gamma-\mathrm{CD}$ 